rq

إكثار الزيتون بزراعة الأنسجة باستخدام العقل البرعمية

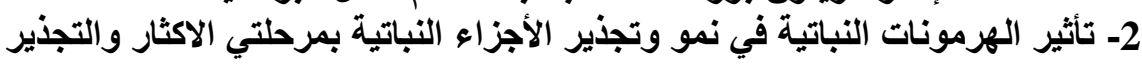

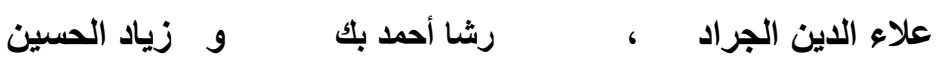

قسم البساتين- كلية الزراعة بدير الزور- جامعة الفرات- سوريا

تضمن البحث اختبار تأثير منظمـات نمو مختلفة على نمو وتجذير الأجزاء النباتيـة من

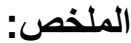

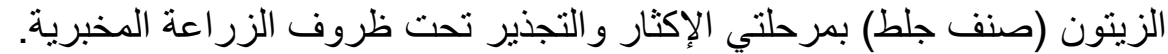

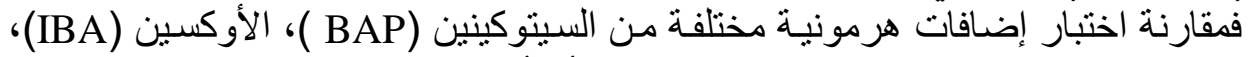

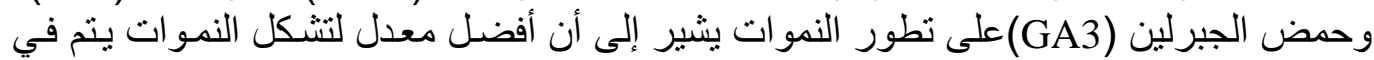

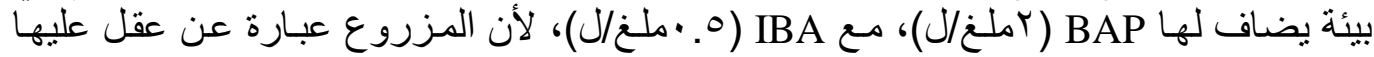

تبين نتائج اختبـار تـأثنير سيتوكينينات مختلفـة (بنزيـل أمينو بيورين، كينيتين، زيـاتين)

بر عمبن.

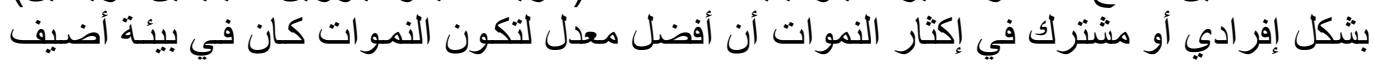

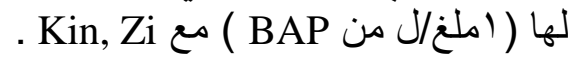

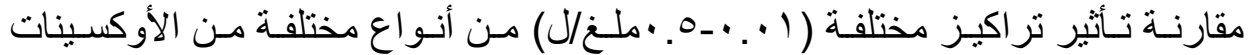

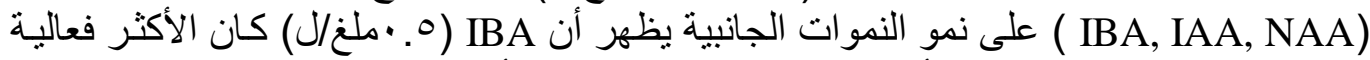

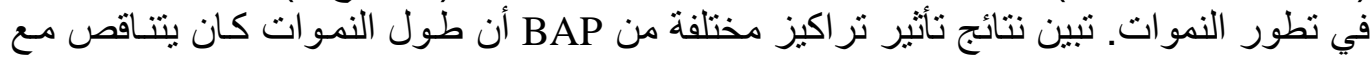

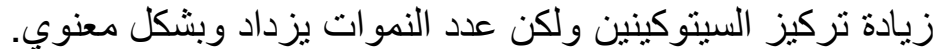

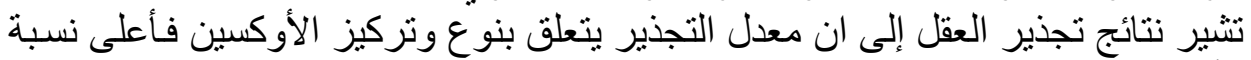

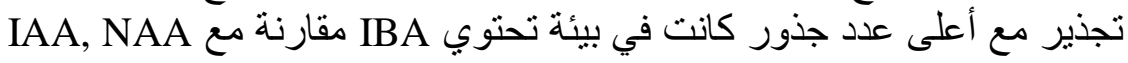
الكلمات المفتاحية: الزينون، زراعة الأنسجة، منظمات النمو، إكثار ، تجذير.

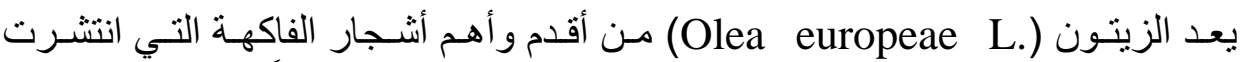

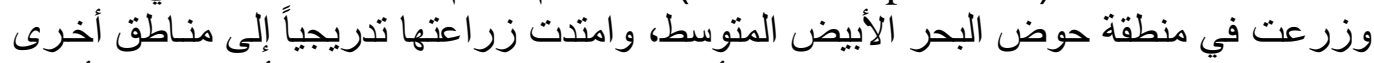

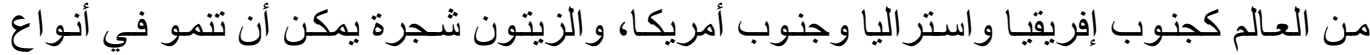

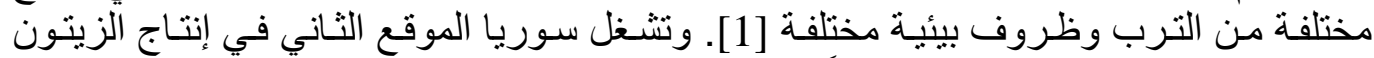

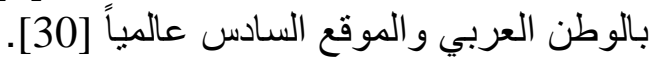

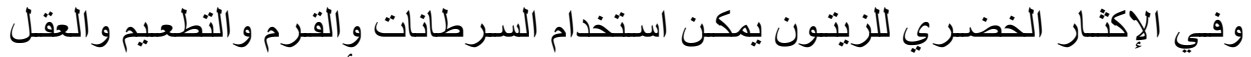
الساقية [2]. يعتبر الإكثار بالعقل الساقية الغضة في الوقت الحاضر هو أكثر الطر ائق الخضر الخضرية

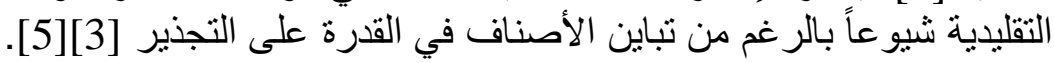

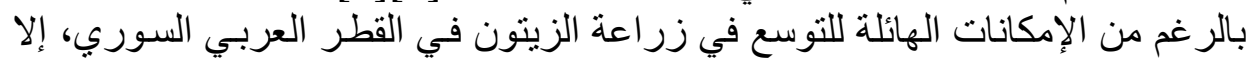

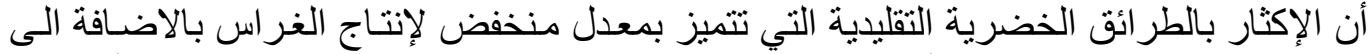

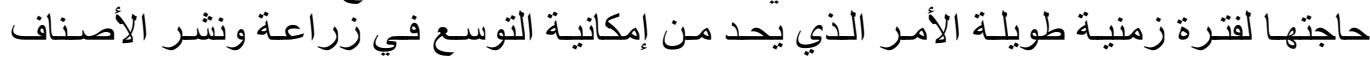

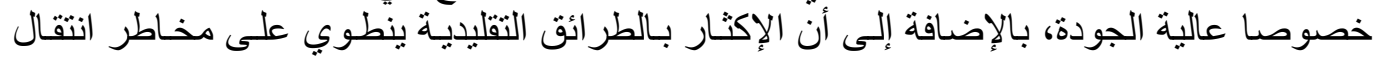

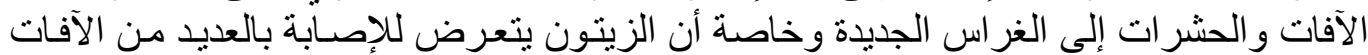

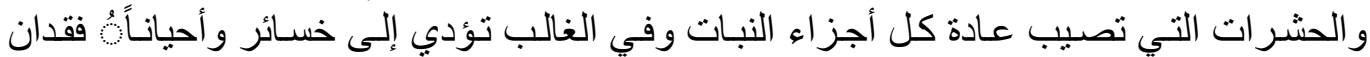
الإنتاج[4].

Fayoum J. Agric. Res. \& Dev., Vol.23, No.1, January, 2009 
r.

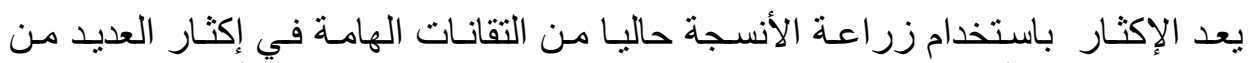

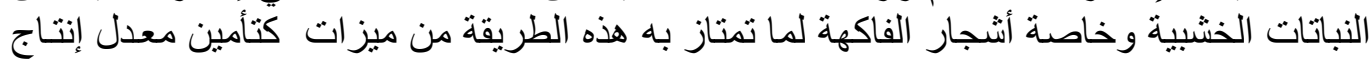

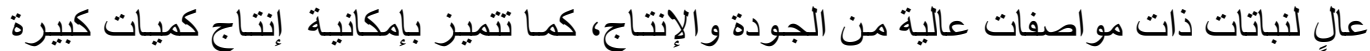

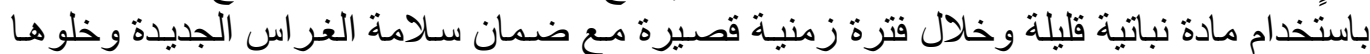

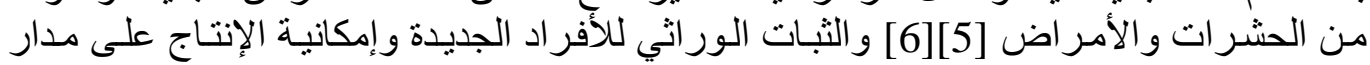

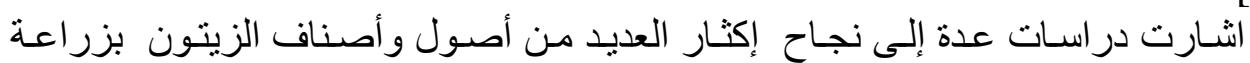

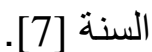

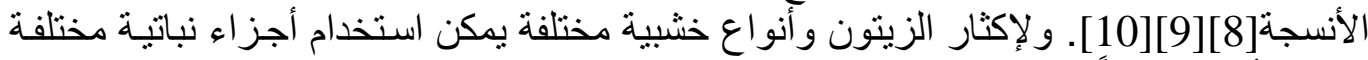

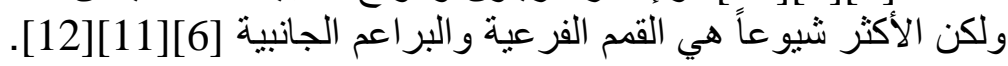

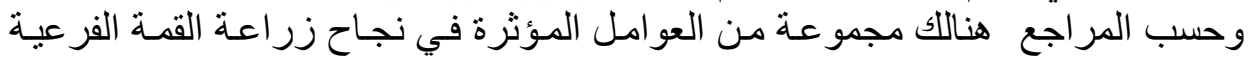

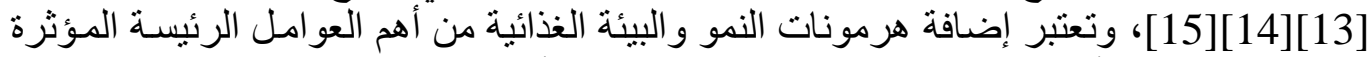

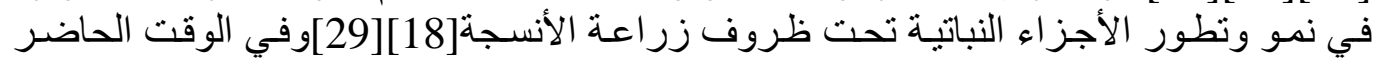

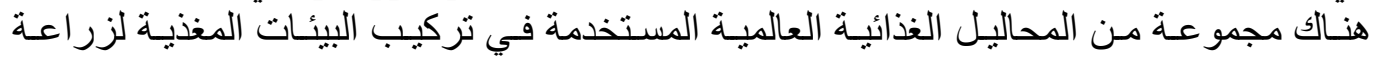

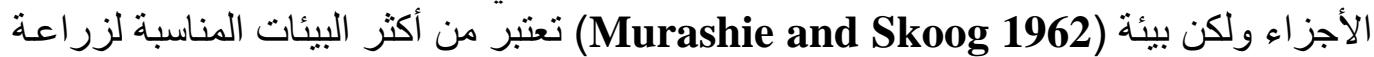

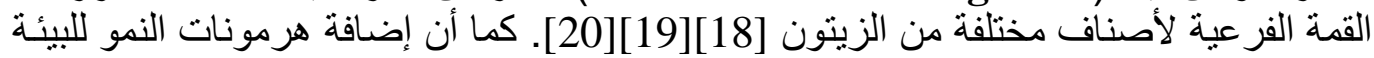

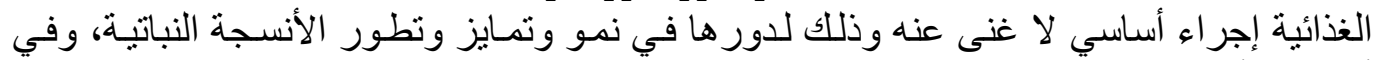

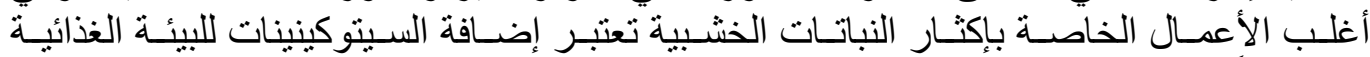

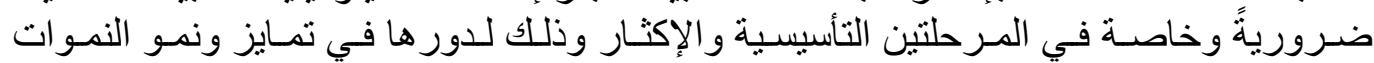

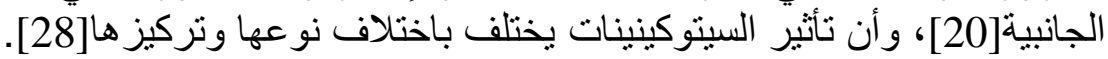

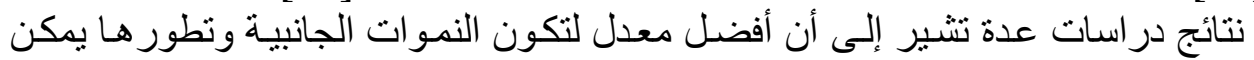

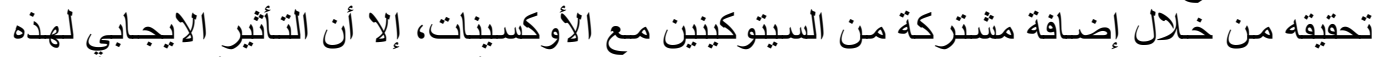

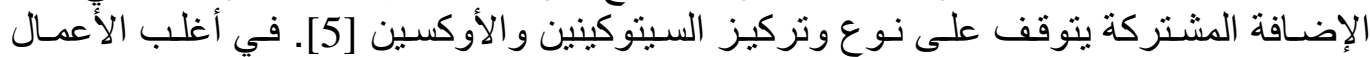

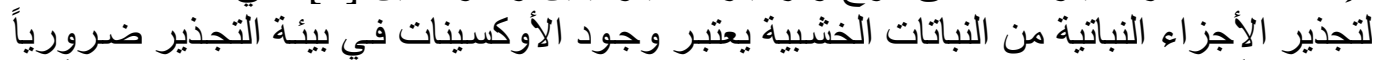

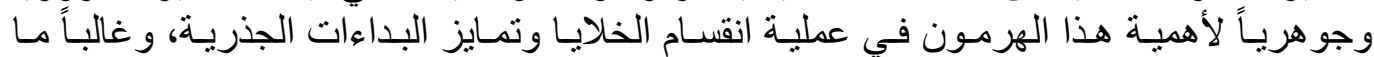
يتوقف تشكل الجذور ونوعيتها على نوع وتركيز الأوكسين المستخدم [21].

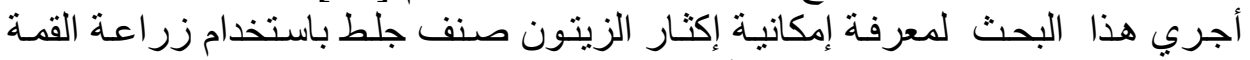

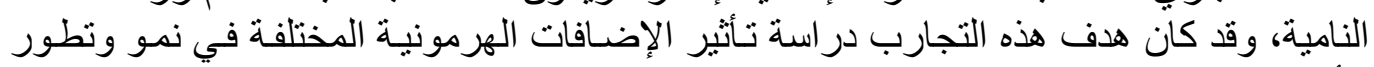

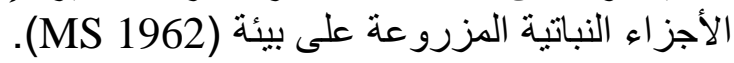

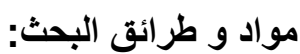
ا - مكان و تاريخ تنفيذ البحث:

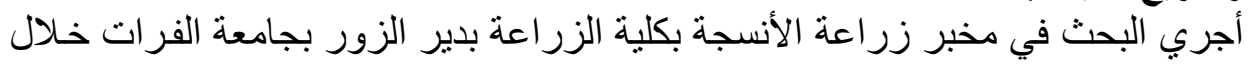

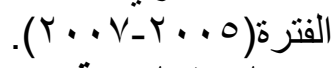

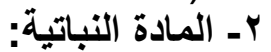

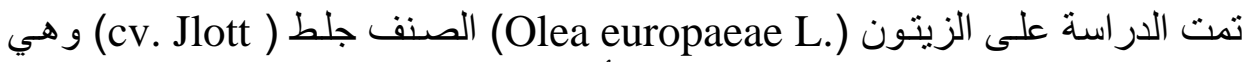

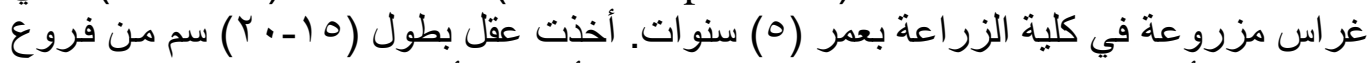

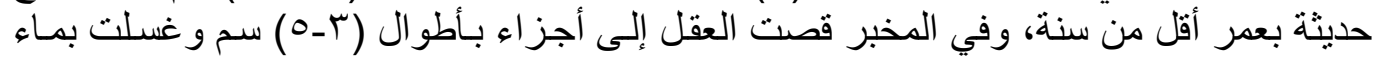

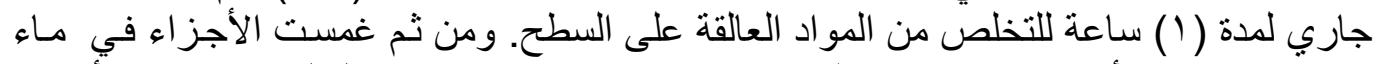

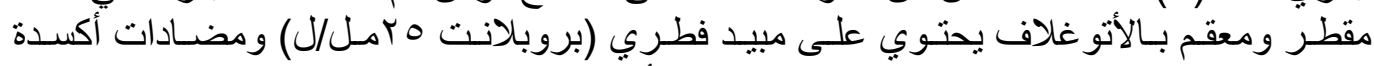

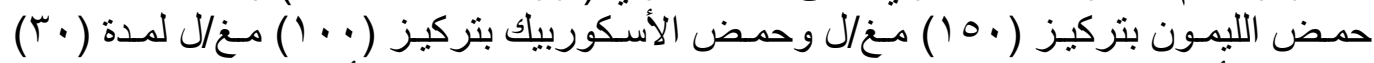

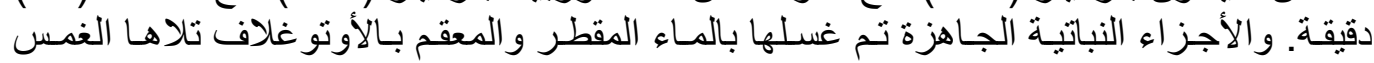

Fayoum J. Agric. Res. \& Dev., Vol.23, No.1, January, 2009 
r

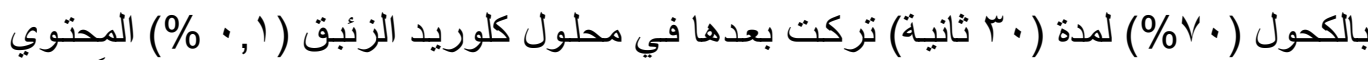

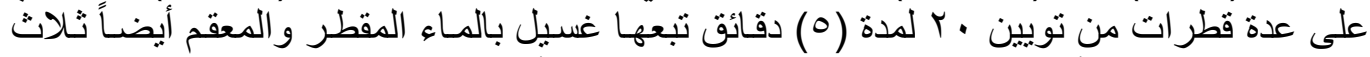
مر ات، ثُم زرعت الأجزاء المطهرة على البيئة الغذائية في أنابيب الاختبار.

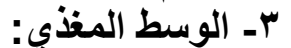

في كل الاختبار ات استخدمت بيئة غذائبة أساسية تكونت مـن العناصـر المعدنيـة الكبرى

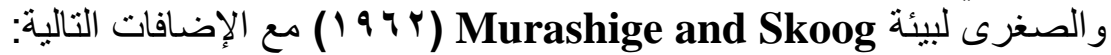

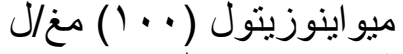

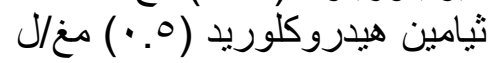

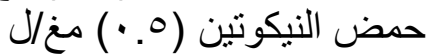
بيرودوكسين هيدروكلوريد (0. (•) ) مغ/ل)

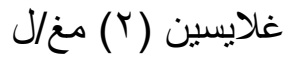

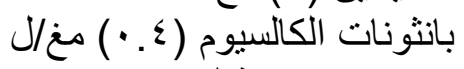

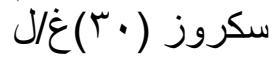

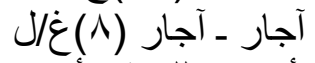

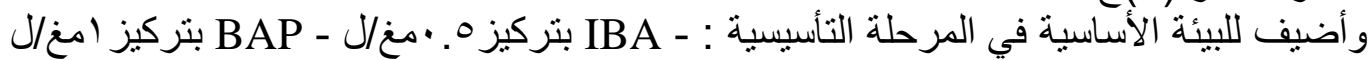

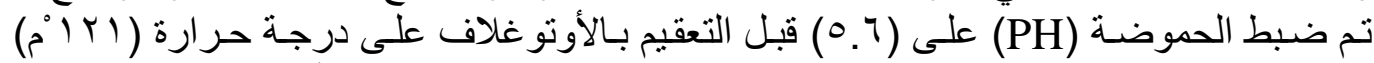

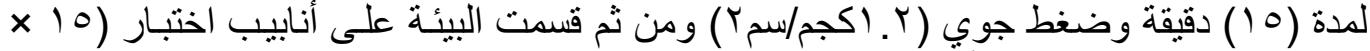

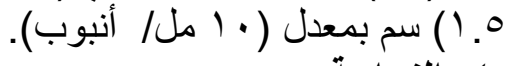

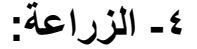

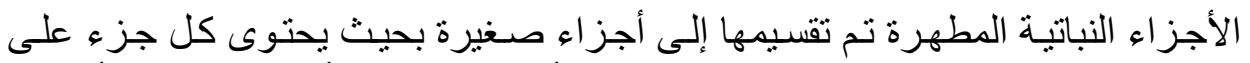

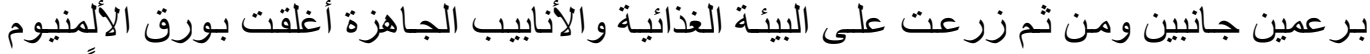

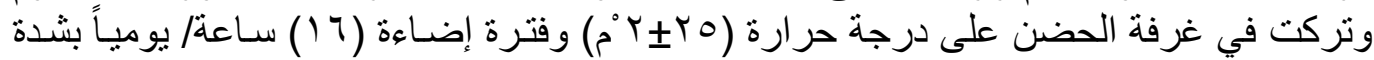

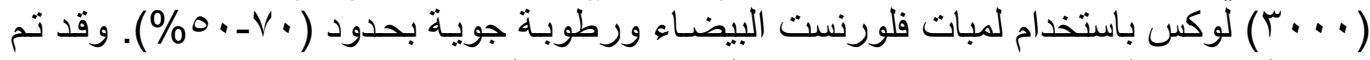

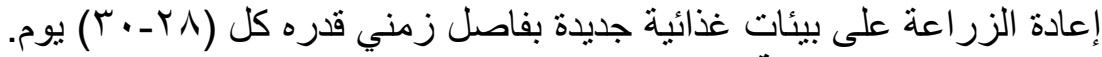

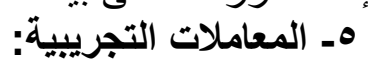

تم اختبار النقاط النالية في التجارب: * إخبى مرحلة الإكثار:

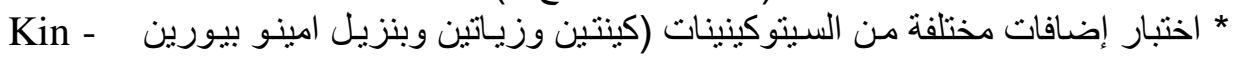
BAP-Zi

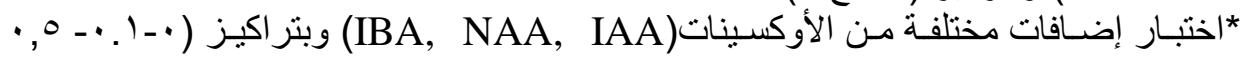

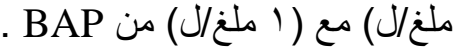

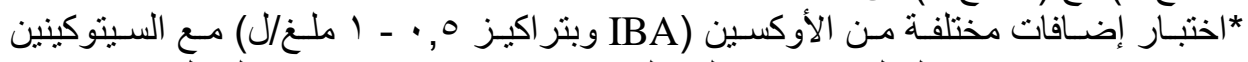

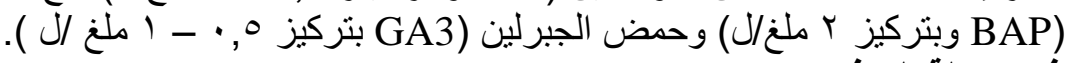

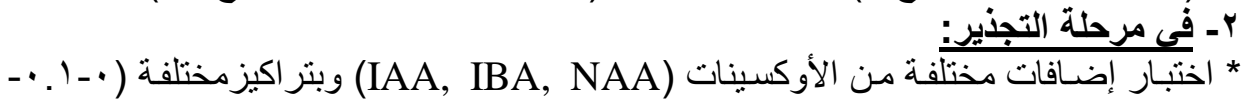
(ملغ/ل)

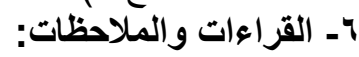
تم تدوين القراءات و الملاحظات حسب المرحلة كالنالي:

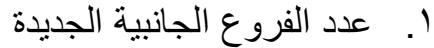
r r آ. طول الفروع الجانبية الجديدة

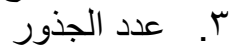

Fayoum J. Agric. Res. \& Dev., Vol.23, No.1, January, 2009 


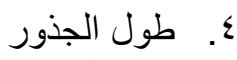

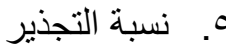

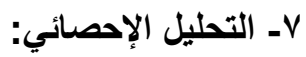

نفذت التجارب بتصميم القطاعات العثو ائية الكاملة ونم تحليل التباين باستخدام البرنـامج

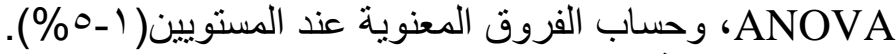

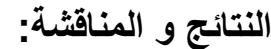

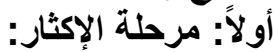

ـ ت تأثير إضافات هرمونية مختلفة:

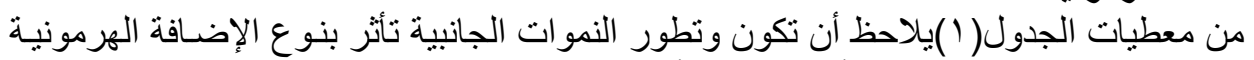

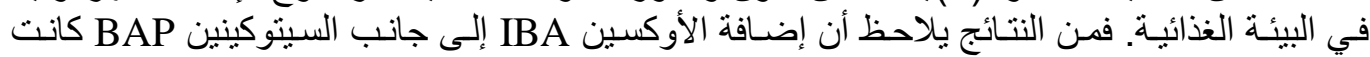

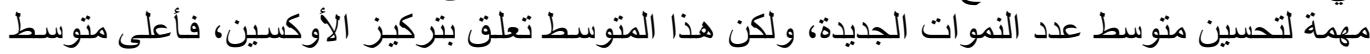

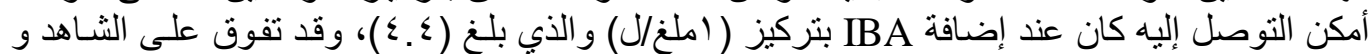

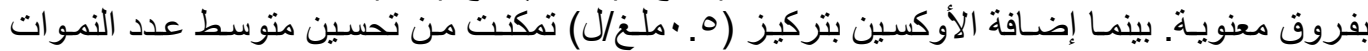

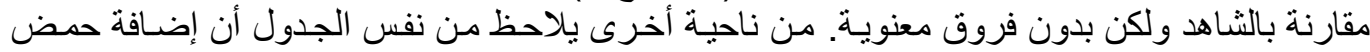

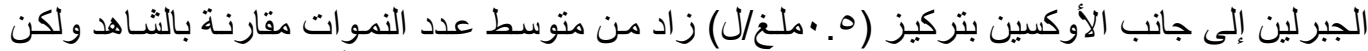

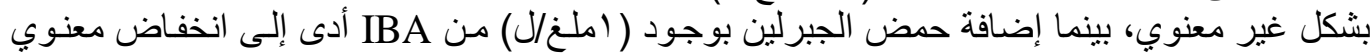

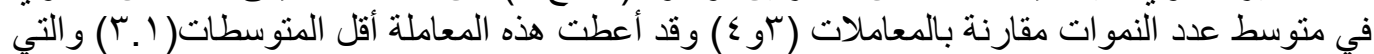
لم تختلف عن الثاهد معنوياً.

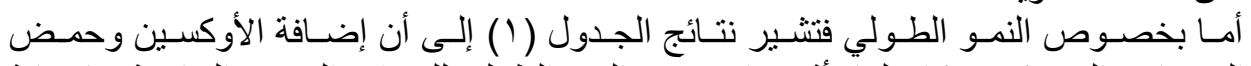

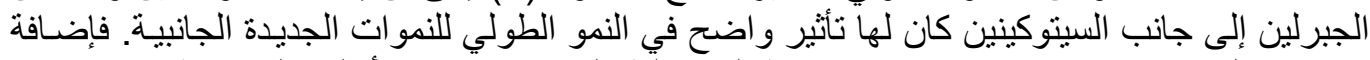

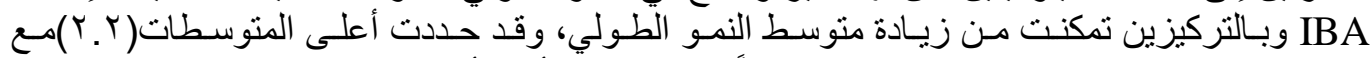

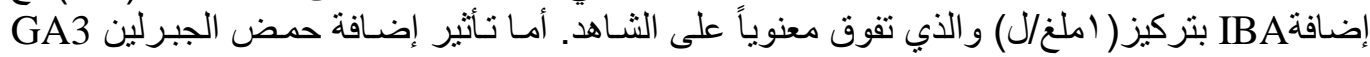

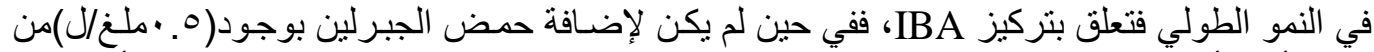

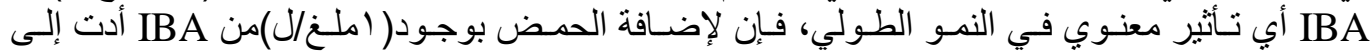

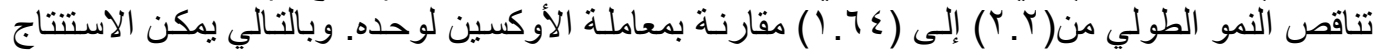

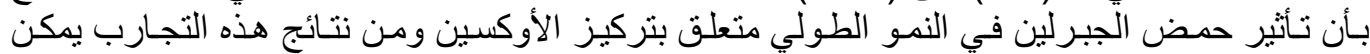

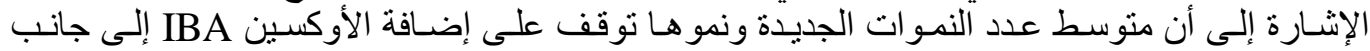

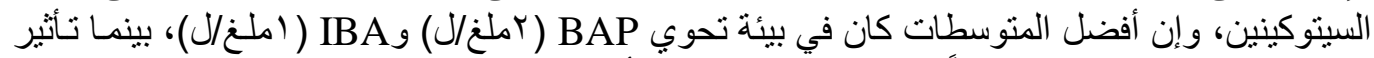

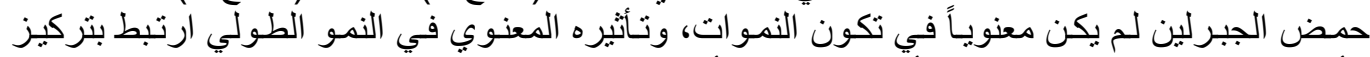

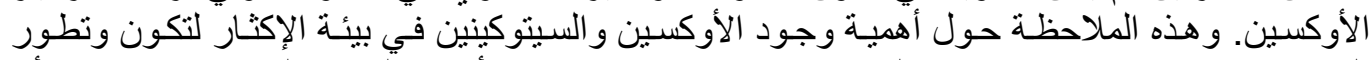

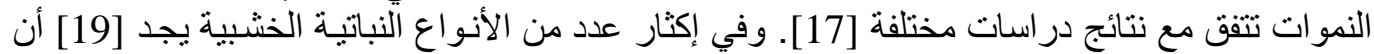

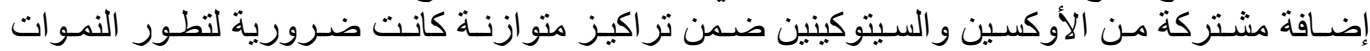

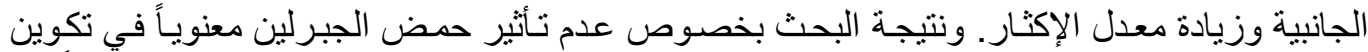

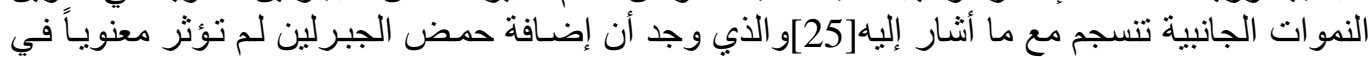

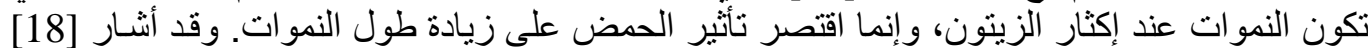

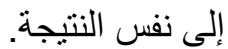

جدول (1) تأثير إضافات هرمونية مختلفة في نمو النموات الجانبية من الزيتون صنف جلط

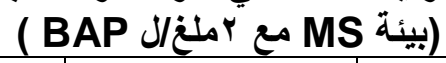

\begin{tabular}{|c|c|c|c|}
\hline متوسط طول النموات & متوسط ع عدد النموات & $\begin{array}{l}\text { GA3 } \\
\text { مغ/J }\end{array}$ & $\begin{array}{l}\text { IBA } \\
\text { مغ/J }\end{array}$ \\
\hline 0.84 & 3.3 & 0 & 0 \\
\hline 1.35 & 3.6 & 0 & 1 \\
\hline
\end{tabular}

Fayoum J. Agric. Res. \& Dev., Vol.23, No.1, January, 2009 
$r r$

\begin{tabular}{|c|c|c|c|}
\hline 1.83 & 4.2 & $\mathbf{1}$ & $\mathbf{1}$ \\
\hline 2.2 & 4.4 & $\mathbf{0}$ & $\mathbf{0 . 5}$ \\
\hline 1.64 & 3.1 & $\mathbf{1}$ & $\mathbf{0 . 5}$ \\
\hline 0.804 & 1.399 & \multicolumn{2}{|c|}{$\mathbf{1 \% \text { LSD }}$} \\
0.595 & 1.036 & \multicolumn{2}{|c|}{$\mathbf{\%}$} \\
\hline
\end{tabular}

ـ تأثير أنواع و تراكيز مختلفة من الأوكسينات:

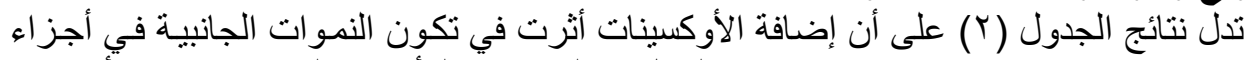

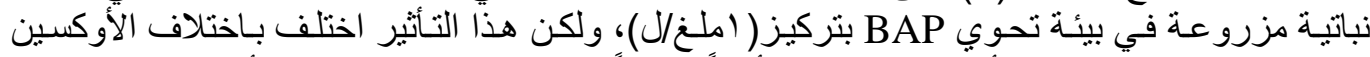

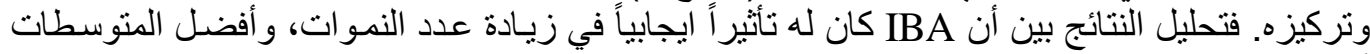

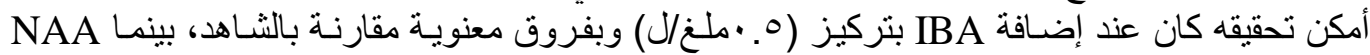

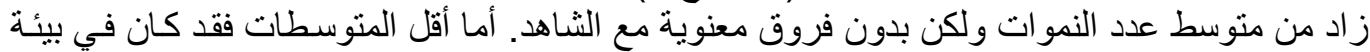

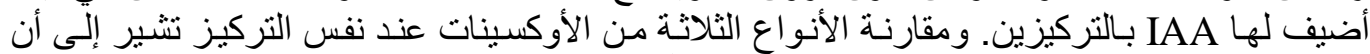

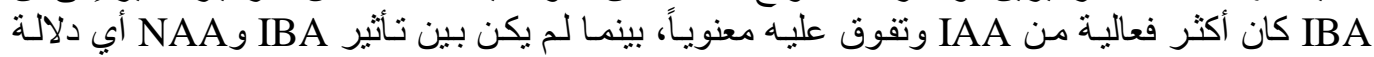

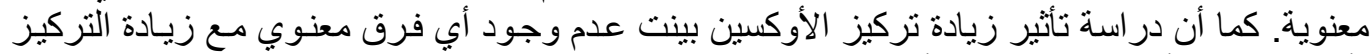

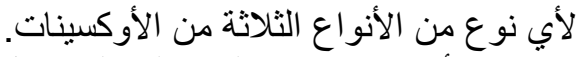

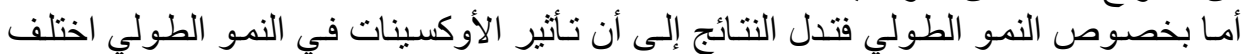

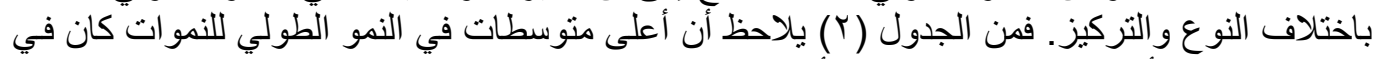

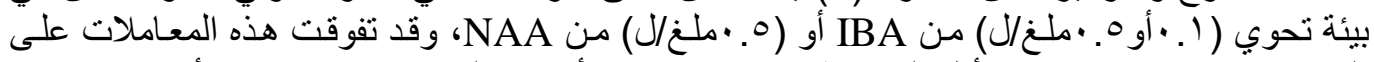

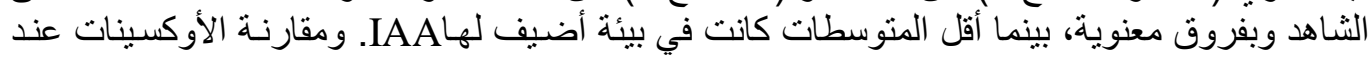

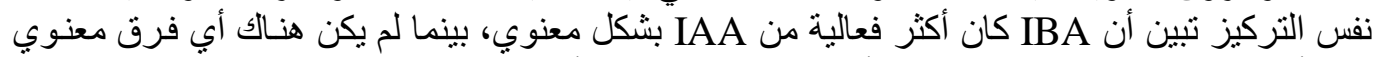

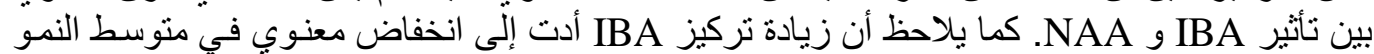

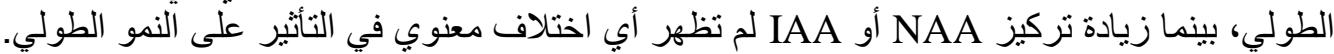

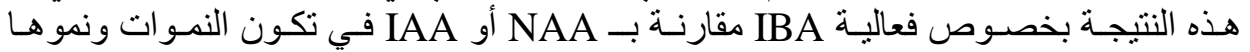

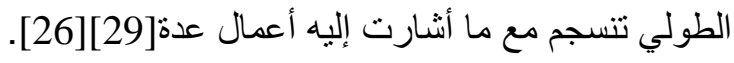

جدول(Y) تأثير تراكيز وأنواع مختلفة من الأوكسينات في نمو النموات الجانبية من الزيتون صنف جلط

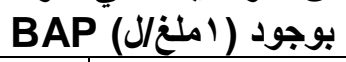

\begin{tabular}{|c|c|c|}
\hline متوسط طول النموات & متوسط عدد النموات & المعاملة \\
\hline 0.729 & 1.66 & BAP \\
\hline 1.03 & 2.58 & $\begin{array}{c}\text { BAP } \\
\text { IBA } 0.1\end{array}$ \\
\hline 1.308 & 2.83 & $\begin{array}{c}\text { BAP } \\
\text { IBA } 0.5\end{array}$ \\
\hline 0.908 & 2.25 & $\begin{array}{c}\text { BAP } \\
\text { NAA } 0.1\end{array}$ \\
\hline 1.08 & 2.33 & $\begin{array}{c}\text { BAP } \\
\text { NAA } 0.5\end{array}$ \\
\hline 0.791 & 1.5 & BAP \\
\hline
\end{tabular}

Fayoum J. Agric. Res. \& Dev., Vol.23, No.1, January, 2009 
rs

\begin{tabular}{|c|c|c|} 
& & \multicolumn{1}{|c|}{ IAA $\mathbf{0 . 1}$} \\
\hline 0.883 & 1.75 & $\begin{array}{c}\text { BAP } \\
\text { IAA } \mathbf{0 . 5}\end{array}$ \\
\hline 0.315 & 1.188 & $\mathbf{1} \%$ LSD \\
0.234 & 0.880 & $\mathbf{5 \%}$ \\
\hline
\end{tabular}

ـ ت تأثير تراكيز مختلفة من السيتوكينين:

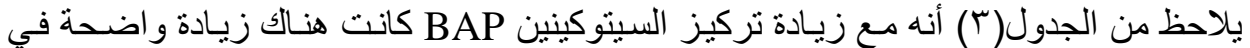

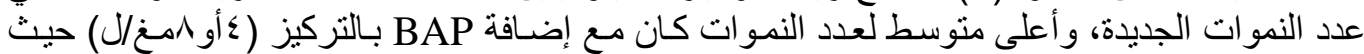

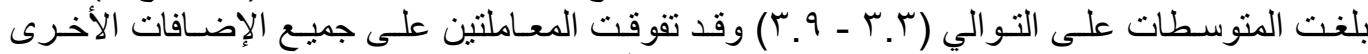

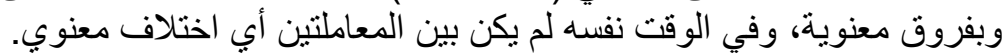

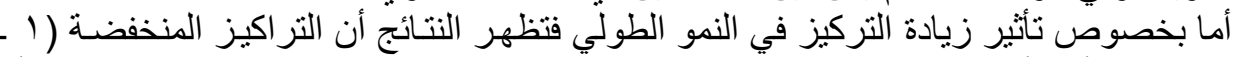

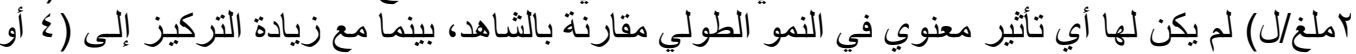

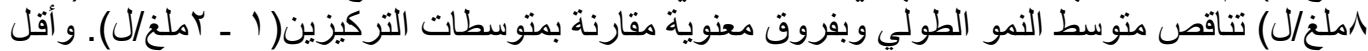

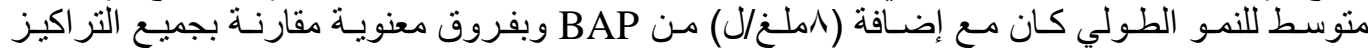

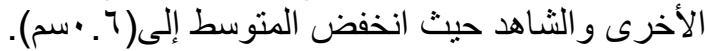

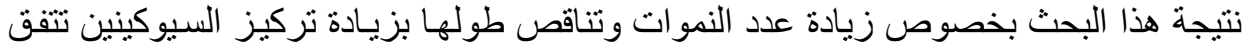

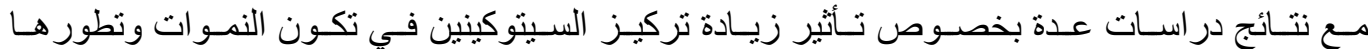

[23][26][25]. وبشكل عام يجد [5][12] أن اختيار البيئة ونوع الإضافة من منظمات النمو وتركيزهـا

يتوقف على صنف الزيتون.

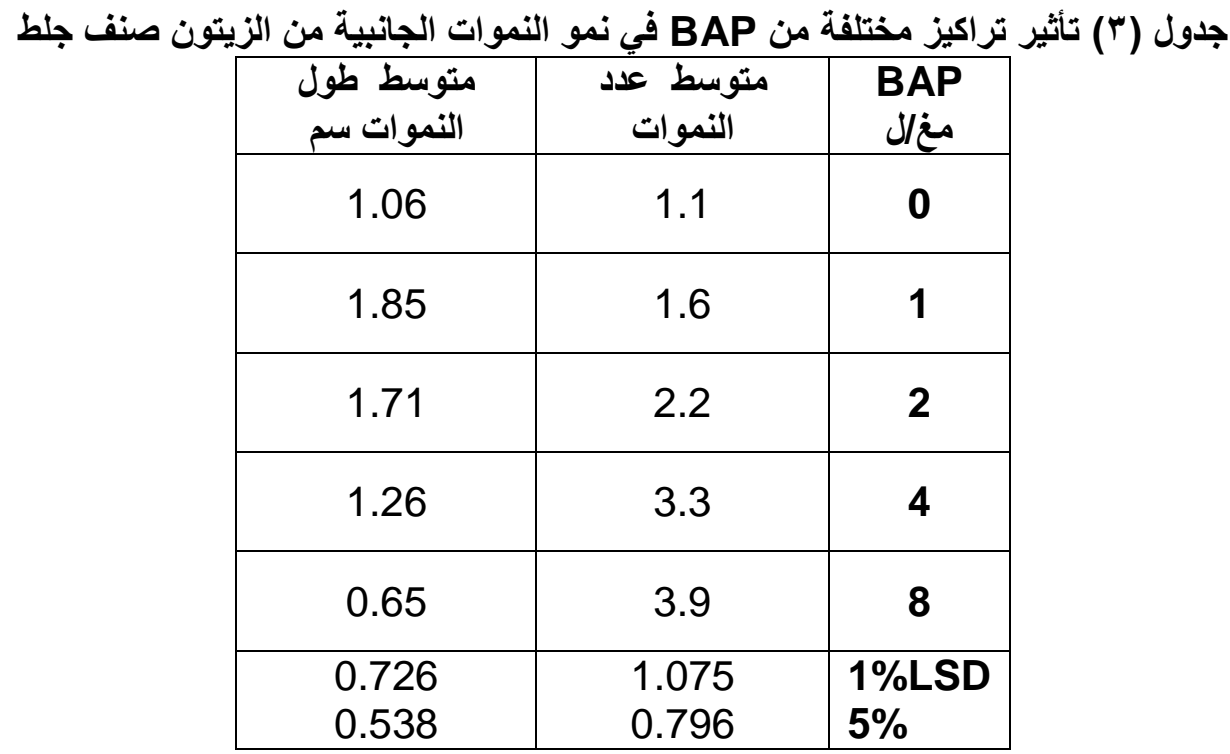

Fayoum J. Agric. Res. \& Dev., Vol.23, No.1, January, 2009 


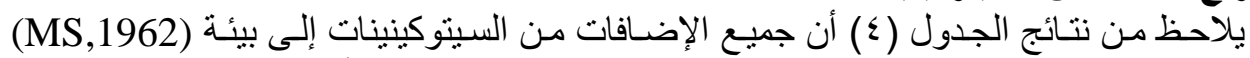

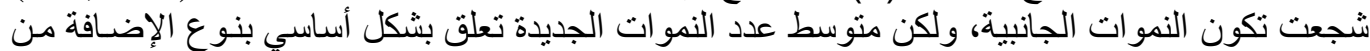

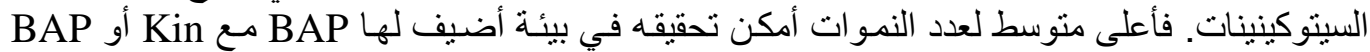

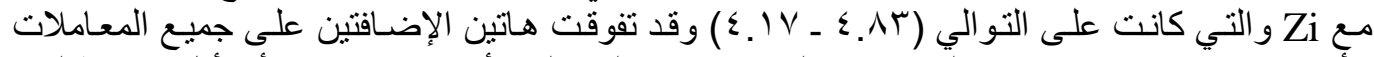

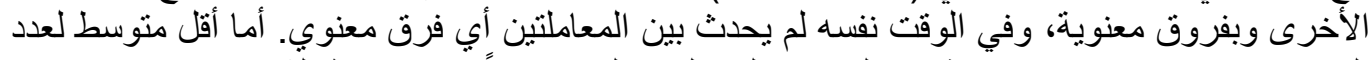

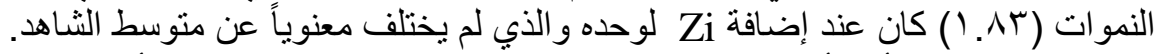

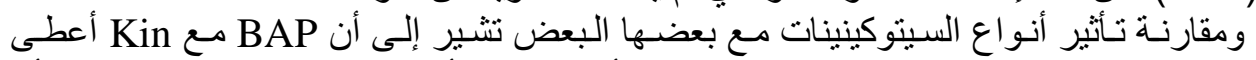

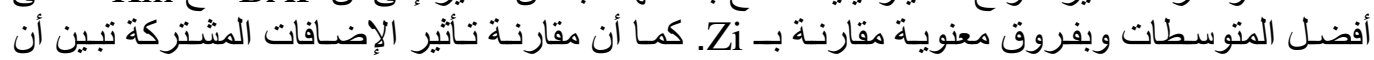

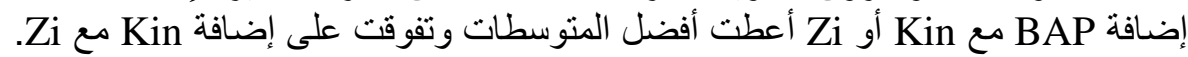

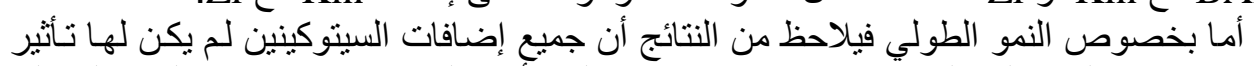

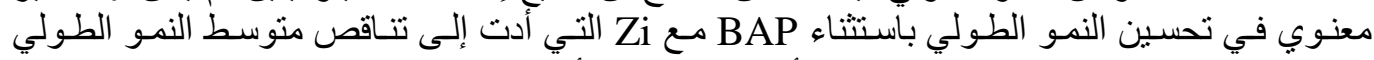

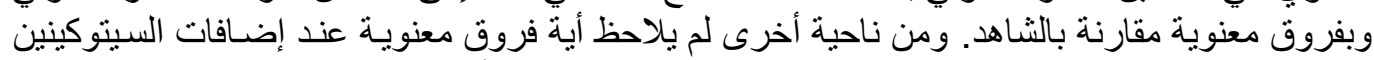

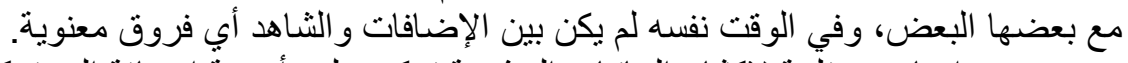

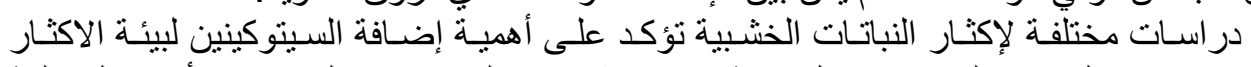

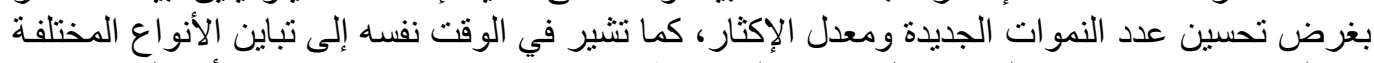

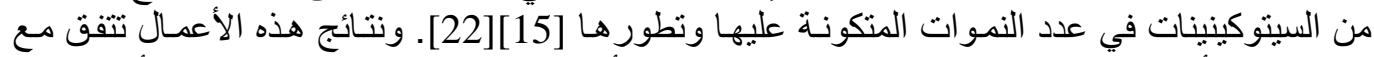

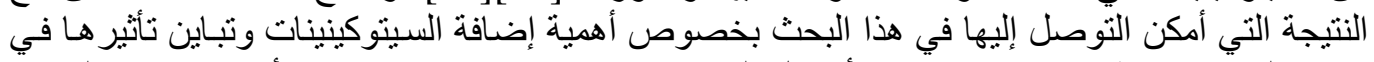

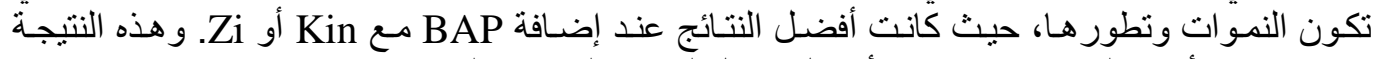

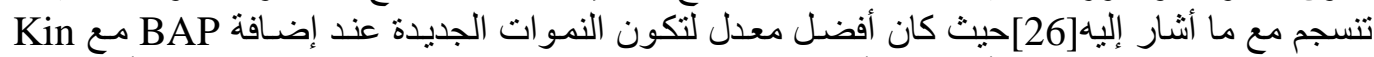

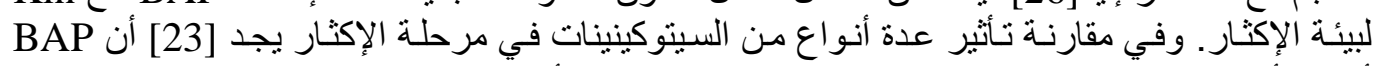

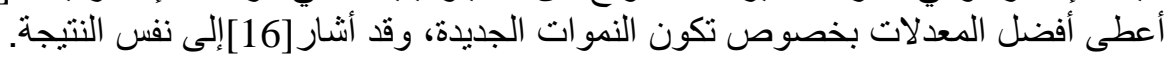

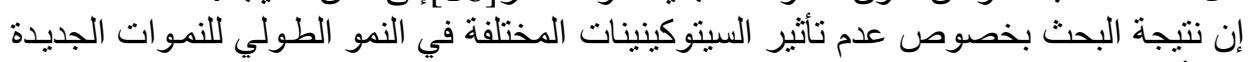

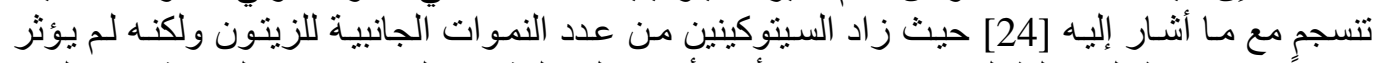

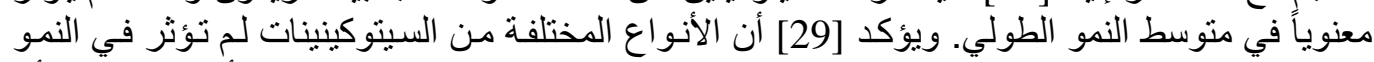

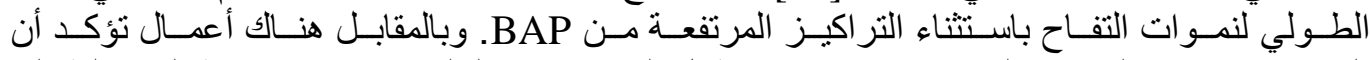

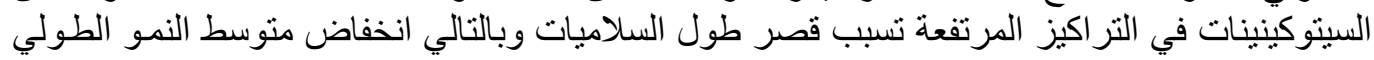

\begin{tabular}{|c|c|c|}
\hline \multicolumn{3}{|c|}{ صنف جلط ( ملغ/ل) } \\
\hline متوسط طول النموات سم & متوسط عدد النموات & المعاملة \\
\hline 1.83 & 1.08 & الثاهد \\
\hline 1.53 & 2.58 & BA \\
\hline 1.35 & 2.25 & Kin \\
\hline 1.78 & 1.83 & $\mathbf{Z i}$ \\
\hline 1.2 & 4.83 & BA+Kin \\
\hline 1.15 & 4.16 & $B A+Z i$ \\
\hline 1.42 & 2.5 & Kin+Zi \\
\hline
\end{tabular}

Fayoum J. Agric. Res. \& Dev., Vol.23, No.1, January, 2009 
rq

\begin{tabular}{|l|l|l|} 
& & \\
\hline 0.875 & 1.420 & $\mathbf{1} \%$ LSD \\
0.648 & 1.052 & $\mathbf{5 \%}$ \\
\hline
\end{tabular}

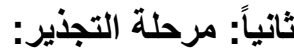

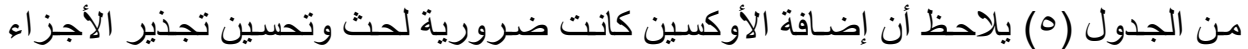

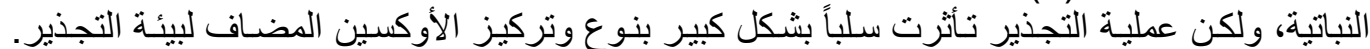

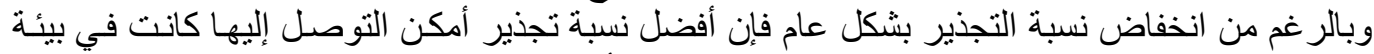

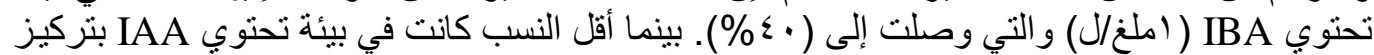

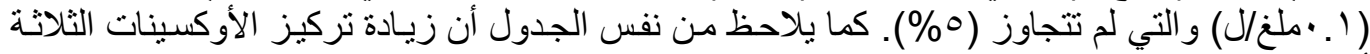

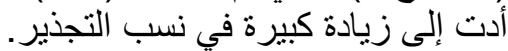

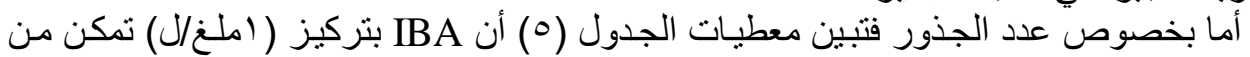

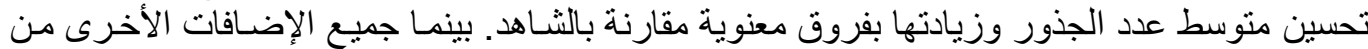

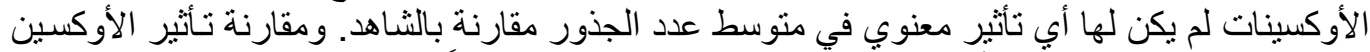

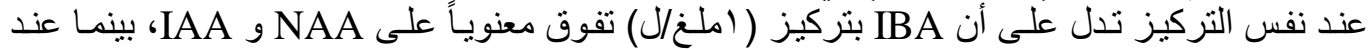

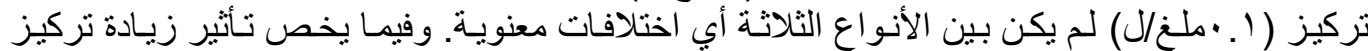

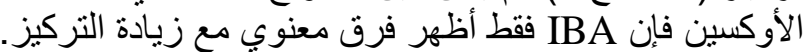

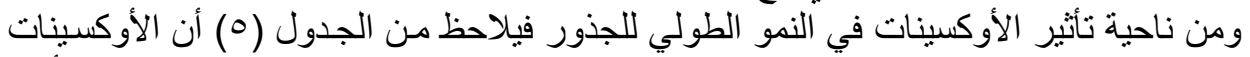

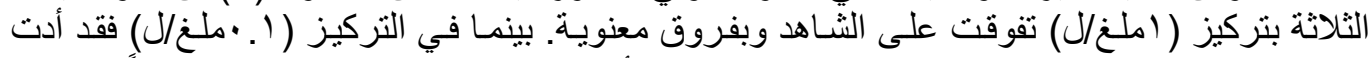

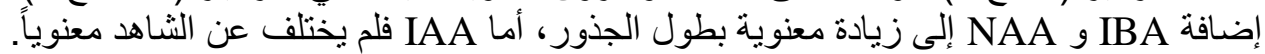

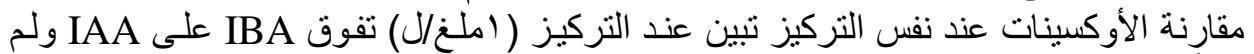

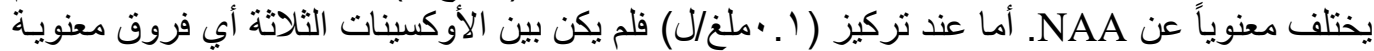

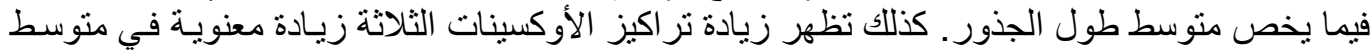

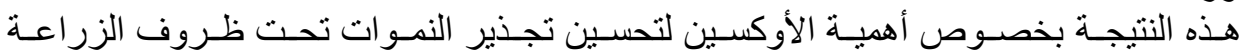

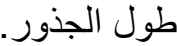

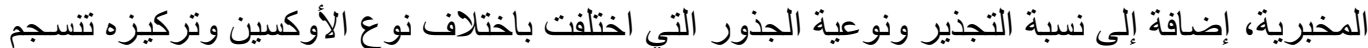

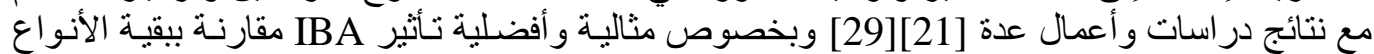

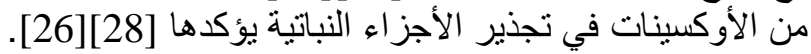

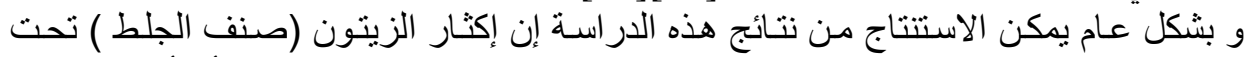

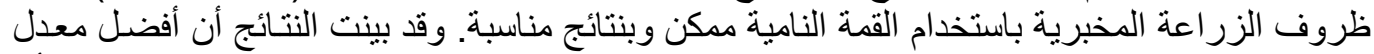

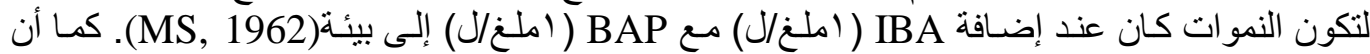

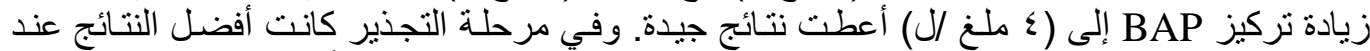

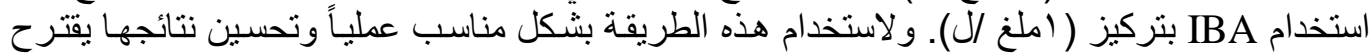

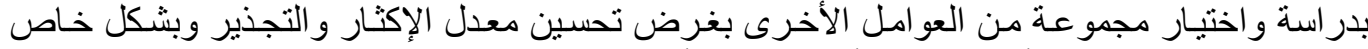
اختيار العو امل الخاصة بتأثير النبات الأم و تر اكيب أخرى من البيئة الغذائية.

جدول (•) تأثير أنواع و تراكيز مختلفة من الأوكسينات في تجذير الأجزاء النباتية من الزيتون صنف جلط من الاوكينات

\begin{tabular}{|c|c|c|c|}
\hline نسبة التجذير\% & متوسط طول الجذور & متوسط عدد الجذور & الأوكسين \\
\hline 3 & 0.71 & 1.08 & 0 \\
\hline 15 & 1.4 & 1.08 & $\begin{array}{l}\text { IBA } \\
0.1\end{array}$ \\
\hline
\end{tabular}

Fayoum J. Agric. Res. \& Dev., Vol.23, No.1, January, 2009 
rV

\begin{tabular}{|c|c|c|c|}
40 & 2.25 & 1.66 & $\begin{array}{c}\text { IBA } \\
\mathbf{1}\end{array}$ \\
\hline 15 & 1.45 & 1.16 & $\begin{array}{c}\text { NAA } \\
\mathbf{0 . 1}\end{array}$ \\
\hline 30 & 2.08 & 1.25 & $\begin{array}{c}\text { NAA } \\
\mathbf{1}\end{array}$ \\
\hline 5 & 0.91 & 1.16 & $\begin{array}{c}\text { IAA } \\
\mathbf{0 . 1}\end{array}$ \\
\hline 20 & 1.66 & 1.25 & $\begin{array}{c}\text { IAA } \\
\mathbf{1}\end{array}$ \\
\hline & 0.570 & $\begin{array}{l}0.486 \\
\mathbf{1} \% \text { LSD } \\
\mathbf{5}\end{array}$ \\
\hline
\end{tabular}

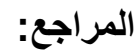

1- NAJIBA B., A.ABOUSALIM D., WALAILOUDIYI E., BENALI D., 2003Effect of Culture Medium on Micro propagation of Olive(Olea europaea L.)cv. Moroccan Picholinee. Biotechnol. Agron. Soc. Environ, 2003 7(3-4), 177-182.

2- ZUCCHINI M., DEAGAZIO M., 2004- Micro propagation of Olive Cultivar from Germplasm Preservation. Publisher: Springer Sciencet Business Media B.V., P: 589-592.

3- ROBERT K. 1997- IN VITRO PROPAGATION OF ENDANGERED WOODZ PLANT SPECIES AND CULTIVARS AS A CONTRIBUTION TO THE CONSERVTION OF GENETIC RESOURCES. Jahresbericht .Bo.26 06. Institut feur Biologie. Wiesbaden.10 P.

4- RUGINI E. 1986- Olive (OLea europaea L.). In: Bogag, Y.P.S. (ED.), Biotechnology in Agriculture and Forestry Trees, vol(1). Springer, Heidelberg, pp: 253-267.

5- SANTOS N., BRITO G., PINTO G. 2003- In vitro Plantlet Regeneration of Olea europaea ssp. Maderensis. Sci. Hort, 97(1), 83-87.

6- KHAN M., RASHID H., QURAISHI A., 2002- In Vitro Shoot Development from juvenile Cuttings of Field Growth Olive (Olea europaea L.) cv. Leccino. J. Bio. Sci, 2(7), 438-440.

7- DREW R., 1995- Application of Biotechnologyto Fruit and Nut Species. The sixth confer. Australasian. Lismore. NSW. 11-15 Sept.

8- LEVA R., PETRUCCELLI R., MULEO R., GORETTI R., BARTOLINI G. 1995- Influenza Di Fattori Trofici, Regolativie Condizioni Del Meezo Nutritivo Sulla Coltura in Vitro Di Diverse Cultivar Di Olive. Rende (CS). 26-28 gennaio, 239-248.

9- GONZALES-RIO F., GURRIARAN M.J., REVILLA M., 1994- Desiccation and Cryoperesrvation of Olive (Olea europaea L.) Embryos. Gryo. Lett, 15, 337-342.

10- RUGINI E. 1990- In Vitro Culture of The Olive: An Overview of The Present Scientific Status. Avta. Hort, 286, 93-96.

Fayoum J. Agric. Res. \& Dev., Vol.23, No.1, January, 2009 
rᄉ

11- RAHMAN M.M., AMIN M., AHMED R. 2004- In Vitro Rapid Regeneration from Cotyledon Explant of Native-Olive (Elaeocarpus robustus Roxb.). Asian J. plant. Sci., 3(1), 31-35.

12- SHIBLI R., SHATNAWI M., AL-JABOORY K., 2001- Somatic Embryogenesis and Plant Recovery from Callus of Nabali Olive (Olea europaea L.). Sci. Hort, 88, 243-256.

13- STEFANO B., SIGFRIDO R., 1999- In Vitro Olive Shoot Regeneration as Affected by Different Hormone Treatments. Orto floro fruttit, 57, 406-413.

14- GARICA-BERENGUER A., DURAN-GONZALES R., 1990- Mineral Media for In Vitro Propagation of Juvenile "picual" Microcuttings. Acta. Hort. 286: In ter. Symp. On olive growing. Abstract.

15- BRHADDA N., ABOUSALIM A., LOUDITI DE., BENALI D. 2003- Effect of Culture Medium on Micro Propagation of Olive (Olea europaea L.) cv. Moroccan Picho-Line. Bio. Agron. Soc. Envi, 7(3-4), 177-182.

16- SANKHLA, D., T-D Davis., Sankhla N., 1996- In Vitro Regeneration of Silk Tree (Albizza julibrissin) from Excised Roots. Plant. Cell. Tiss. And Organ culture. 44, 83-86.

17- NICOLETTA F., FAMIANI F.,PROIETTI P., STANICA F., 1996Influence of Growth Regulators and Light on In Vitro Shoot Regeneration in M26 Apple Rootstock. J. Hort. Sci. 71(6): 859-865.

18- DIMASSI-THERIOU K., 1994- In Vitro Propagation of "Kalamon" Olives(Olea europaea L.). Adv. Hort. Sci. 8:185-189.

19- BORNMAN C.H., 1983- Possibilities and Constraints in the Regeneration of Tree from Cotyledonary Muddles of Piea abies In Vitro. Physiol. Plant. pp: 57-116.

20- DREW R A., 1991- In Vitro Culture of Adult and Juvenile Explants of Passiflora Species. Plant. Cell. Tiss and Org. Cult. 26:23-27.

21- NEVILLE A.,M. BINNS., D CLOUTIER., 1995- Auxins Salt Concentrations and their Interactions During In Vitro Rooting of Winter Hardy and Hybrid Tea Roses. Hort. Sci. 30(7): 1436-1440.

22- PATTNAIK S., Y SAHOOD., P CHAND., 1996- Micropropagation of a Fruit Tree Morus australis Poir. Plant. Cell. Rep. 15:841-845.

23- AISH M., H RASHID., I HUSSAIN., 2007- Propagation Rate Effects of BAP and Kinnetin on Banana (Musa ssp. AAA Group)"Basrai". Hort. Sci. 42(1): 10-46.

24- RAMA P., C.A PONTIKINS., 1990- In Vitro Propagation of Olive (Olea europaea sativa L.) Kalamon. J. Hort. Sci. 65: 347-353.

25- BATROLINI G., A.R LEVA., A BENELLI., 1990- Advances in In Vitro Culture of Olive: Propagation of cv. Maurino. Acta. Hort., 286: 41-44.

26- RAHMAN M.M., M.N AMIN., S AHMAD., 2003- Rapid Clonal Propagation of Native-Olive(Elaeocarpus robustus Roxb.)Using Tissue Culture Technique. J. Bio. Sci., 3: 1107-1113.

Fayoum J. Agric. Res. \& Dev., Vol.23, No.1, January, 2009 
27- KHAN M.R., H RASHID., A QURAISHI., 2002- Development of Aseptic in Field-Grown Olive(Olea europaea L.)cv. Pendollino. Asian J. Pl. Sci., 3: 220-221.

28- COZZA R., D TURCO., C.B BATI., M.B BITONI., 1997- Influence of Growth Medium on Mineral Composition and Leaf Histology in Micropropagation Plantiets. Plant. Cell. Tiss. Org. Cult. 51: 215-223.

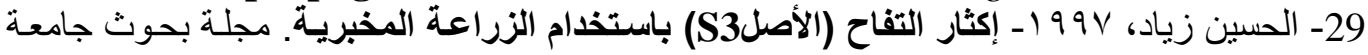

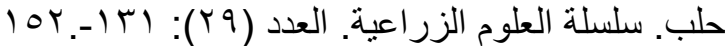

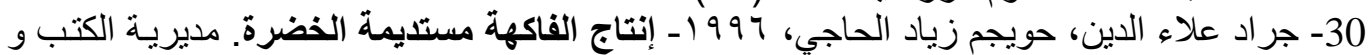

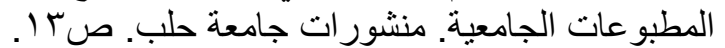

\section{Tissue Culture Propagation of Olive (Olea europaea L.) cv. Jlott by Using Shoot Tip: \\ 2-Effect of Different Hormones on The Growth and Rooting of The Explants in the Multiplication and Rooting Phases Ala din Jarad Z.Al-Hussin}

Rsha A. Beik

Dep. of Horticulture-Faculty of Agriculture-Al-Furat University- Syria.

\section{ABSTREACT}

The aim of this experiment was to study the effect of various grow the regulators on shoot tip regeneration and rooting of Olive (Olea europaea L.) cv. Jlott in the multiplication and rooting phases.

Different phytohormones (BAP, IBA, GA3) tested for shoot development. The best shoot formation resulted on medium supplemented with 2 mg/l BAP (benzylaminopurine) and 0,5 mg/L IBA (Indol Bioyric Acid).

Various cytoKinnins (BAP, Kinnetin, Zeatin) either singly or in combination were examined for their influence on shoot multiplication. The results showed that the adventitious shoot formed best on medium with $1 \mathrm{mg} / \mathrm{L}$ of BAP and Kinn. or Zi.

Among different concentrations $(0.1-0.5 \mathrm{mg} / \mathrm{L})$ and type of auxins (IBA, NAA, IAA) tested for shoot growth, IBA $(0,5 \mathrm{mg} / \mathrm{L}$ affected the shoot regeneration significantly. Shoot height was reduced with increasing the concentration levels of BAP, but number of shoots per explants was significantly increased. 
$\varepsilon$.

The results showed that the rooting rate was significantly deponded upon auxin type and its concentration. The highest rate of rooting with maximum number of roots occurred in medium containing IBA compard to NAA or IAA.

Key word: Olive, Tissue culture, Growth regulators, Multiplication, Rooting. 